\title{
Condiciones de trabajo y morbilidad entre mineros del carbón en Guachetá, Cundinamarca: la mirada de los legos
}

\author{
Claudia P. Jiménez-Forero ${ }^{1,2}$, Ivonne T. Zabala², Álvaro J. Idrovo ${ }^{3}$ \\ 1 Programa de Maestría en Salud Ocupacional y Ambiental, Escuela de Medicina y Ciencias de la Salud, \\ Universidad del Rosario, Bogotá, D.C., Colombia \\ 2 Facultad de Relaciones Internacionales, Estrategia y Seguridad, Universidad Militar, Bogotá, D.C., Colombia \\ 3 Departamento de Salud Pública, Escuela de Medicina, Facultad de Salud, Universidad Industrial de Santander, \\ Bucaramanga, Colombia; Programa de Fisioterapia, Universidad Manuela Beltrán, Bucaramanga, Colombia
}

Introducción. En Colombia, la investigación sobre las condiciones de trabajo y salud en la minería carbonífera es escasa y no toma en consideración la percepción de la población expuesta y sus comportamientos frente a los riesgos inherentes.

Objetivo. Determinar la asociación entre las condiciones de trabajo y la morbilidad percibidas por los trabajadores de minas de carbón en Guachetá, Cundinamarca.

Materiales y métodos. Se hizo un estudio transversal con 154 trabajadores seleccionados aleatoriamente del total registrado en la alcaldía municipal. Se indagó sobre las características sociodemográficas, y las condiciones de trabajo y de salud en las minas. Se estimaron las prevalencias de los trastornos respiratorios, osteomusculares y auditivos, y se exploraron las asociaciones entre algunas condiciones de trabajo y los eventos adversos con prevalencia superior al $30 \%$ de forma bivariada y múltiple mediante regresiones de Poisson con varianza sólida.

Resultados. Los trabajadores eran, en su mayoría, hombres, con edades entre los 18 y los 77 años. Los problemas de salud más frecuentemente reportados fueron: dolor lumbar $(46,1 \%)$, dolor miembros superiores (40,3\%), dolor en miembros inferiores $(34,4 \%)$, trastornos respiratorios $(17,5 \%)$ y problemas auditivos (13,6\%). Se registraron diferencias importantes en la percepción, dependiendo de la antigüedad laboral y las condiciones de trabajo, subterráneo o de superficie.

Conclusión. Los riesgos más reconocidos fueron los relacionados con los trastornos osteomusculares, por ser más cercanos en el tiempo con respecto al trabajo realizado ("descuento temporal"). Se proponen acciones basadas en la identificación de rasgos psicológicos, para mejorar la percepción del riesgo entre los mineros del carbón.

Palabras clave: condiciones de trabajo, morbilidad, minas de carbón, salud ocupacional, percepción social, Colombia.

doi: http://dx.doi.org/10.7705/biomedica.v35i0.2439

\section{Work conditions and morbidity among coal miners in Guachetá, Colombia: The miners' perspective}

Introduction: Investigations in Colombia about work and health conditions in coal mining are scarce and few have focused on the perception of the exposed population and their behaviors in response to inherent risks.

Objective: To determine the association between work conditions and the perception of morbidity among coal miners in Guachetá, Cundinamarca.

Materials and methods: A cross-sectional study was performed with 154 workers selected randomly from the total registered with the municipality. Information about social and demographic characteristics and work and health conditions in the mines was gathered. The prevalence was estimated for respiratory, musculoskeletal and auditory disorders. The associations between certain work conditions, and events with a prevalence over $30 \%$ were explored using bivariate and multivariate analyses with Poisson regressions with robust variance.

Results: Workers were mostly men. Ages ranged from 18 to 77 years. Most frequently reported health problems were: back pain (46.1\%), pain in an upper limb (40.3\%), pain in a lower limb (34.4\%), and

\footnotetext{
Contribución de los autores:

Claudia P. Jiménez-Forero e Ivonne Tatiana Zabala: concepción y diseño del estudio

Claudia P. Jiménez-Forero y Álvaro J. Idrovo: análisis e interpretación de los datos

Todos los autores participaron en la escritura del manuscrito.
} 
respiratory $(17.5 \%)$ and auditory problems (13.6\%). Significant differences in perception were found depending on time on the job and underground or ground work conditions.

Conclusions: The most recognized risks were those associated with musculoskeletal disorders since they were closer in time to the work performed (time discount). Some actions to identify psychological traits are proposed in order to improve risk perception among coal miners.

Key words: Working conditions, morbidity, coal mining, occupational health, social perception, Colombia.

doi: http://dx.doi.org/10.7705/biomedica.v35i0.2439

En las últimas décadas, los modelos económicos han favorecido la explotación del carbón en países en desarrollo como Colombia (1). Esto se hace evidente en diversos informes en los que el país resulta ser atractivo para los inversionistas extranjeros, gracias al incremento de la seguridad y pese a las incertidumbres generadas por políticas ambientales cada vez más exigentes $(2,3)$. A pesar de la importancia de la extracción carbonífera en Colombia, las estadísticas sobre enfermedad laboral y accidentes de trabajo en el sector no son confiables y se supone que existe una gran subestimación, sobre todo por la informalidad con la que se trabaja en un número significativo de minas $(4,5)$.

La investigación sobre las condiciones de trabajo y la salud en los sitios de minería carbonífera y sus alrededores, es escasa a nivel nacional. Por un lado, están los estudios de higiene industrial que han empezado a brindar información sobre el grado de exposición a material en partículas (6-9); otro grupo de investigadores se ha dedicado a evaluar los efectos citogenéticos en humanos $(10,11)$ y animales $(12-15)$. Por su parte, la mayoría de los estudios epidemiológicos han sido descriptivos, dejando de lado la exploración de asociaciones que podrían permitir acciones que promuevan mejoras en el ambiente laboral. Por ejemplo, hay estudios que exploran globalmente las condiciones de trabajo y de salud $(5,16)$, las características socioeconómicas y de riesgo laboral (17), así como la salud respiratoria de los mineros de socavón $(18,19)$. En los estudios de asociación, se ha reportado una mayor prevalencia de asma entre los menores de edad residentes en las cercanías de las minas (20) y de neumoconiosis entre quienes se dedican a las actividades con

Correspondencia:

Claudia P. Jiménez-Forero, Universidad del Rosario, Carrera 24 № 63C-69, tercer piso, Bogotá, D.C., Colombia

Teléfonos: (300) 567 7551; (571) 4635260

clalien2003@hotmail.com

Recibido: 02/07/14; aceptado: 19/03/15 mayor exposición a material en partículas (21), la capacidad de predicción de la espirometría y la oximetría para detectar el daño pulmonar (22), y algunos factores asociados con las alteraciones osteomusculares (23). La mayoría de los estudios han adoptado una aproximación técnica (la visión de los 'expertos'), sin considerar la percepción de la población expuesta y sus comportamientos frente a los riesgos inherentes al trabajo en las minas de carbón (visión de los 'legos').

Existen atributos individuales y sociales que determinan la percepción del riesgo e influyen en la forma en que se actúa frente a él. Es así como, la definición de riesgo, su potencial de generar daño, el tiempo entre la exposición y la manifestación del daño, el origen antropogénico o natural, la distribución equitativa de riesgos y beneficios en la sociedad, sus efectos potenciales sobre las generaciones futuras, la capacidad de control, la familiaridad, la habituación, la exposición voluntaria o no, y los valores ambientales, entre otros, se relacionan con la percepción del riesgo (24-27).

En este contexto, el objetivo de este estudio fue explorar la asociación entre la percepción de las condiciones de trabajo y morbilidad entre los trabajadores de las minas de carbón de Guachetá, con el fin de recabar información sobre la forma en que ellos comprenden o representan su actividad laboral. Se espera, así, obtener evidencia científica que apoye las acciones necesarias para mitigar los impactos ocasionados por la minería del carbón entre los trabajadores.

\section{Materiales y métodos \\ Contexto del estudio}

Guachetá está ubicado a $118 \mathrm{~km}$ al norte de Bogotá. La explotación de carbón allí se inició hace aproximadamente 40 años, con unidades empresariales de tipo familiar; posteriormente, se instalaron empresas de mayor envergadura que generaron una gran demanda de mano de obra no calificada, lo cual provocó la migración de trabajadores desde otras regiones. Esto determina 
que un porcentaje significativo de la población trabajadora minera sea flotante. Actualmente, el sector se caracteriza por la proliferación de pequeñas empresas de tipo familiar con explotación rudimentaria, lo que genera altos costos para los dueños debido a la profundidad cada vez mayor de la explotación, las tarifas de la energía eléctrica y el precio del producto en el mercado.

\section{Modelo conceptual del estudio}

El estudio partió de aceptar la validez e importancia del conocimiento de los trabajadores para la adopción de decisiones que promuevan la prevención de eventos adversos asociados con el trabajo. Se trata, entonces, de un estudio enmarcado en enfoques metodológicos como la 'epidemiología popular' (28), la aproximación ecosistémica en salud (29) y la investigación participativa basada en la comunidad (30), los cuales se han utilizado exitosamente en estudios previos (31).

Este tipo de enfoque contrasta con el de la epidemiología tradicional, que prefiere las mediciones objetivas y otorga poco valor al reporte dado por los propios individuos sujetos a una condición o exposición, pues se considera que este puede ser sesgado o, por lo menos, adolecer de errores de medición (32). Si bien esto puede tener impacto en la exploración causal de los factores de riesgo, el conocimiento que los sujetos de estudio tienen sobre los problemas de salud que ellos mismos enfrentan constituye un recurso fundamental y complementario del enfoque tradicional, que permite adoptar intervenciones orientadas a prevenir la exposición o las situaciones adversas para la salud (33). Además, su desconocimiento puede conllevar que los involucrados no acepten las intervenciones porque no corresponden a su cultura (34).

\section{Diseño y participantes}

Se hizo un estudio transversal con trabajadores de las minas de carbón de tres veredas de Guachetá. Mediante muestreo aleatorio simple, se seleccionó una muestra representativa de la población minera trabajadora $(n=787)$, a partir de la base de datos del 2012 suministrada por la alcaldía municipal. Los trabajadores así seleccionados fueron ubicados en sus lugares de trabajo y, en muy pocos casos, en sus hogares; a continuación se les invitó a participar y se recolectaron los datos. El tamaño de la muestra fue de 144 mineros, considerando una prevalencia esperada de $13 \%$, un alfa de 0,05 , un poder de 0,90 y una frecuencia alterna de $23 \%(35)$.
Los criterios de inclusión fueron ser mayor de 18 años de edad y estar laborando en una mina.

Este estudio fue aprobado por el Comité Central de Investigaciones de la Universidad Militar Nueva Granada, como parte del proyecto "Diagnóstico y caracterización de las condiciones laborales de cierta población minera del Municipio de Guachetá".

\section{Recolección de los datos}

Antes de proceder a la recolección de los datos, se usaron tres instrumentos. El primero fue uno de origen español reconocido internacionalmente como idóneo para la identificación de las condiciones de trabajo y de salud (36), del cual se eliminaron algunas preguntas por carecer de relevancia para el estudio o por usar un lenguaje desconocido para los trabajadores del sector. El instrumento original, que corresponde a una visión lega de las condiciones de trabajo y de salud, incorpora las siguientes dimensiones: condiciones de empleo, condiciones de trabajo, amenazas y violencias en el trabajo, y estado de salud (36). De los 85 ítems originales, en el estudio se incluyeron 41 que eran aplicables a las condiciones de trabajo en las minas en estudio.

El segundo instrumento recurrió a preguntas explícitas sobre la percepción del riesgo y la morbilidad sentida, también desde la perspectiva de una visión lega. El tercer instrumento permitió recolectar información a partir de la observación directa de las condiciones de trabajo registrada por los profesionales en salud laboral.

Estos dos últimos instrumentos se formularon en el marco de un ejercicio académico de revisión bibliográfica y de una visita de acercamiento a diferentes minas del municipio de Lenguazaque (Cundinamarca) y de Sogamoso (Boyacá), que sirvieron como referencia a condiciones de trabajo, similares y esperables. Posteriormente, los instrumentos se sometieron al juicio de expertos en salud ocupacional, higiene y minería.

Los problemas de salud explorados se pueden clasificar en respiratorios, auditivos y osteomusculares, los cuales se seleccionaron por ser los más frecuentes entre los mineros del carbón (37), y porque podían explorarse con el diseño de estudio seleccionado y el tamaño de muestra disponible. Los problemas respiratorios incluyeron trastornos respiratorios, neumonía y diagnóstico de acumulación de polvo de carbón en los pulmones (posible neumoconiosis); entre los problemas 
auditivos se indagó por las afecciones y alteraciones auditivas diagnosticadas, en tanto que entre los problemas osteomusculares se incluyeron el dolor de espalda, el dolor en miembros superiores, el dolor en miembros inferiores y la enfermedad osteomuscular diagnosticada. Se usaron términos populares de fácil comprensión para los mineros en lugar de términos médicos, con el fin de garantizar una mejor comunicación entre trabajadores e investigadores.

Antes de las salidas de campo, se entrenó a nueve estudiantes del Semillero de Investigación del programa de Administración de la Seguridad y Salud Ocupacional de la Universidad Militar Nueva Granada en la utilización de los instrumentos; estos mismos estudiantes participaron en la recolección de los datos.

Se hizo un primer viaje al municipio para hacer la prueba piloto a un grupo de trabajadores, los cuales no fueron incluidos en la muestra. El trabajo de campo se llevó a cabo en cinco jornadas, en las que se utilizaron los instrumentos entre los trabajadores seleccionados que aceptaron participar en el estudio mediante firma de consentimiento informado escrito y autorización verbal registrada en archivo de audio.

Para la observación directa de las condiciones de trabajo, se ingresó a la bocamina con un guía después de obtener el consentimiento verbal del dueño y se hicieron in situ los análisis de seguridad estructural y de gases. El ingreso a la mina se suspendía en caso de que se estuvieran adelantando labores de voladura o mantenimiento del inclinado principal, o se presentara inundación.

\section{Métodos estadísticos}

En un primer momento se describieron las variables categóricas mediante porcentajes y, las cuantitativas, mediante medidas de tendencia central y dispersión según la distribución obtenida con la prueba de Shapiro-Wilk.

Para medir la exposición acumulada, se construyó una matriz con base en el tiempo de trabajo en la mina y la ocupación dentro o fuera de ella, y se tuvo en cuenta que las labores de los 'piqueros', 'frenteros' y 'cocheros' se desarrollan dentro de la bocamina, en tanto que las de los llamados 'malacateros', 'patieros', 'ministros', administradores o jefes de mina, almacenistas y otros, se llevan a cabo en la superficie. Las principales actividades de cada tipo de labor se encuentran resumidas en el cuadro 1.
Las matrices de exposición ocupacional son elementos frecuentes de los estudios de epidemiología ocupacional y sirven para analizar los efectos en la salud asociados con exposiciones durante largos periodos; en general, estas cruzan una o varias variables que relacionan el grado de exposición presente en un momento dado con el tiempo en que ocurre la exposición (38).

A continuación, se compararon los terciles de tiempo en la mina y las ocupaciones mediante la prueba de ji al cuadrado, la prueba exacta de Fisher o la de Kruskal-Wallis. En los casos de las tablas de contingencia con más de cuatro celdas, que requirieron la aplicación de una prueba exacta, se usó el algoritmo descrito por Mehta y Patel (39). Posteriormente, para los problemas de salud con prevalencia superior al $30 \%$, se calcularon las razones de prevalencia con base en las variables sociodemográficas y de condiciones de trabajo y, por último, se hicieron las regresiones de Poisson con varianza sólida (40), con el fin de obtener razones de prevalencia ajustadas. Solo los eventos más frecuentes se analizaron mediante regresiones, pues el reducido tamaño de la muestra no brindaba el poder suficiente para explorar problemas de menor prevalencia. Todos los análisis se hicieron con el programa estadístico Stata $11^{\mathrm{TM}}$ (Stata Corporation, College Station).

\section{Resultados}

Algunas características de los participantes se resumen en el cuadro 2. Como se puede apreciar, la mayoría eran hombres $(94,8 \%)$, con edades entre los 18 y los 77 años (mediana=31,5). El $64,3 \%$ de los participantes provenía de la vereda Santuario, seguidos por el 20, $8 \%$ de participantes procedentes de la vereda Peñas y del 14,9\% de la vereda Rabanal. El tiempo de trabajo en las minas fluctuó entre menos de un año y 46 años (mediana=6 años), lo que varió según la mina donde se hacía la entrevista entre un mes y ocho años (mediana=12,5 meses). El tipo de contratación predominante fue el directo (92,9\%), en tanto que la subcontratación a través de empresas temporales fue baja (1,3\%); sin embargo, 5,8 \% de los trabajadores desconocían la forma de contratación bajo la cual se encontraban vinculados. Cerca de la mitad de los trabajadores (48,0\%) percibía tener estabilidad laboral. La percepción de la seguridad fue de rango medio en $16,9 \%$ de ellos y, baja, en el $13,0 \%$, mientras que el $22,1 \%$ dijeron no tener conocimiento sobre este tema. 
Cuadro 1. Cargos y principales actividades en las minas de carbón estudiadas

\begin{tabular}{|c|c|c|c|c|}
\hline \multirow[t]{2}{*}{$\begin{array}{l}\text { Nombre } \\
\text { del cargo }\end{array}$} & \multicolumn{2}{|c|}{$\begin{array}{l}\text { Lugar de trabajo } \\
\text { principal }\end{array}$} & \multirow[t]{2}{*}{ Responsabilidad en la mina } & \multirow[t]{2}{*}{$\begin{array}{l}\text { Características generales } \\
\text { del puesto de trabajo }\end{array}$} \\
\hline & Profundidad & Superficie & & \\
\hline Frentero & $x$ & & $\begin{array}{l}\text { Abrir el sendero para la } \\
\text { explotación del manto de carbón } \\
\text { e instalar el sostenimiento de la } \\
\text { mina en madera; mantenimiento } \\
\text { del inclinado principal }\end{array}$ & $\begin{array}{l}\text { Trabajo principalmente de pie, manipulación } \\
\text { del martillo neumático, carga y descarga de } \\
\text { maderos, manipulación de explosivos para } \\
\text { voladura }\end{array}$ \\
\hline Piquero & $x$ & & $\begin{array}{l}\text { Responsable de la explotación } \\
\text { del manto de carbón }\end{array}$ & $\begin{array}{l}\text { Trabajo generalmente en posición forzada, } \\
\text { manipulación de martillo neumático, pico } \\
\text { y pala }\end{array}$ \\
\hline Cochero & $x$ & & $\begin{array}{l}\text { Transporte, carga y descarga de } \\
\text { coches con carbón }\end{array}$ & $\begin{array}{l}\text { Trabajo de pie y desplazamiento en la } \\
\text { mina, manipulación de la pala para carga y } \\
\text { descarga del coche, empuje con el cuerpo } \\
\text { del coche metálico de aproximadamente } 1 \\
\text { a } 4 \text { toneladas }\end{array}$ \\
\hline Malacatero & & $x$ & $\begin{array}{l}\text { Activación de la subida y bajada } \\
\text { del coche con carbón o personas }\end{array}$ & $\begin{array}{l}\text { Trabajo en posición sentado, encendido del } \\
\text { motor del malacate, atención a la señal de } \\
\text { subida o bajada del coche }\end{array}$ \\
\hline Patiero & & $x$ & $\begin{array}{l}\text { Selección en el patio del carbón } \\
\text { separado de la roca }\end{array}$ & $\begin{array}{l}\text { Trabajo en posición de pie, desplazamiento } \\
\text { en superficie, manipulación de rastrillo } \\
\text { metálico y pala }\end{array}$ \\
\hline $\begin{array}{l}\text { Ministro, } \\
\text { administrador } \\
\text { o jefe de mina }\end{array}$ & & $x$ & & $\begin{array}{l}\text { Desplazamiento en superficie y } \\
\text { eventualmente en mina, revisión de la } \\
\text { seguridad de los trabajadores y del flujo } \\
\text { de producción }\end{array}$ \\
\hline Almacenista & & $x$ & & $\begin{array}{l}\text { Trabajo en posición sentado y eventualmente } \\
\text { desplazamiento en superficie, clasificación, } \\
\text { almacenamiento, entrega y recepción } \\
\text { de herramientas de trabajo y equipos de } \\
\text { protección personal }\end{array}$ \\
\hline
\end{tabular}

Las ocupaciones más frecuentes fueron las de piquero $(36,4 \%)$, cochero $(18,8 \%)$ y frentero $(14,3 \%)$, trabajadores entre quienes estaban los que pasan la mayoría de la jornada laboral en la profundidad $(69,5 \%)$, lo que se asocia con el hecho de que el 70,1\% de los trabajadores describió su lugar de trabajo habitual como cerrado y 16,9 \% como parcialmente cerrado.

Con respecto a la jornada laboral, todos refirieron trabajar ocho horas diarias; 69,5 \% de los mineros trabajaban de lunes a sábado, 15,6 \%, de lunes a viernes, y solo excepcionalmente los sábados y domingos, o los festivos (feriados). Hubo predominio de los turnos rotatorios (76,0 \%), que incluían el nocturno (31,8\%), el de mañana y tarde (jornada partida) $(26,0 \%)$ y el exclusivamente diurno $(18,2 \%)$.

Según la percepción de la frecuencia de la exposición a condiciones específicas de trabajo durante la jornada laboral, las más relevantes fueron: el ruido $(97,4 \%)$, la respiración de sustancias químicas presentes en el aire en forma de polvo y otras (81,8 \%), así como aquellas condiciones consideradas como la causa de los trastornos osteomusculares, con frecuencias superiores al $61 \%$. Las posiciones corporales adoptadas dependían de la labor desempeñada: los piqueros fueron quienes refirieron con mayor frecuencia tener que adoptar posturas forzadas y contar con menos espacio para hacer los movimientos necesarios o cambiar de postura; los cocheros, frenteros y patieros refirieron trabajar habitualmente de pie, mientras que los malacateros, sentados. Todas estas labores requieren la manipulación de cargas y la realización de movimientos repetitivos.

Al comparar los tres grupos resultantes de la agrupación por los terciles del tiempo laborado en las minas, se observaron diferencias relacionadas con la edad, el sexo (más mujeres en el tercil menor), las actividades subterráneas, la antigüedad en la última mina, la frecuencia de exposición a contaminantes en el aire, las labores con proyección de partículas hacia la cara y el uso o almacenamiento de productos inflamables. 
Cuadro 2. Condiciones de trabajo en las minas bajo estudio de acuerdo con el tiempo de trabajo (terciles) en la minería

\begin{tabular}{|c|c|c|c|c|}
\hline \multirow[t]{2}{*}{ Variables } & \multicolumn{3}{|c|}{ Tiempo de trabajo en las minas (años) } & \multirow[t]{2}{*}{$\mathbf{p}$} \\
\hline & 0 a $4 \quad(n=55)$ & 5 a $10(n=50)$ & 11 a $46(n=49)$ & \\
\hline \multicolumn{5}{|l|}{ Edad (años cumplidos) ${ }^{*}$} \\
\hline 18 a 28 & $23(18-28)$ & $26(19-28)$ & $27(24-28)$ & $0,0001^{\star *}$ \\
\hline 29 a 38 & $33(30-37)$ & $34(29-38)$ & $32,5(29-37)$ & \\
\hline 39 a 77 & $43,5(40-52)$ & $41(39-58)$ & $48,5(39-77)$ & \\
\hline Sexo: hombres & 48 & 49 & 49 & $0,006^{*}$ \\
\hline \multicolumn{5}{|l|}{ Cargo actual } \\
\hline Piquero & 13 & 27 & 16 & $0,003^{*}$ \\
\hline Frentero & 8 & 5 & 9 & \\
\hline Cochero & 16 & 10 & 3 & \\
\hline Patiero & 1 & 2 & 7 & \\
\hline Malacatero & 5 & 2 & 3 & \\
\hline Ministro, supervisor, jefe o administrador & 6 & 1 & 7 & \\
\hline Otro (almacenistas, técnicos) & 6 & 3 & 4 & \\
\hline \multicolumn{5}{|l|}{ Antigüedad (en meses) en la última mina $¥$} \\
\hline 1 a 6 & $5(1-6)$ & $2(1-6)$ & $1,5(1-6)$ & $0,0196 * *$ \\
\hline 7 a 24 & $15(7-24)$ & $18(8-24)$ & $18(9-24)$ & \\
\hline 25 a 96 & $30(30-31)$ & $48(27-60)$ & $36(25-96)$ & \\
\hline \multicolumn{5}{|l|}{ Lugar de trabajo habitual } \\
\hline A la intemperie & 7 & 3 & 12 & $0,023^{*}$ \\
\hline Cerrado & 37 & 43 & 28 & \\
\hline Parcialmente cerrado & 11 & 4 & 9 & \\
\hline \multicolumn{5}{|l|}{ Frecuencia de exposición a tóxicos en aire } \\
\hline Siempre & 25 & 27 & 17 & $0,022^{*}$ \\
\hline Muchas veces & 3 & 4 & 13 & \\
\hline Algunas veces & 12 & 11 & 9 & \\
\hline Solo alguna vez & 2 & 3 & 0 & \\
\hline Nunca & 13 & 5 & 10 & \\
\hline \multicolumn{5}{|l|}{ Frecuencia de manipulación de cargas } \\
\hline Siempre & 25 & 21 & 13 & 0,149 \\
\hline Muchas veces & 5 & 12 & 10 & \\
\hline Algunas veces & 10 & 9 & 16 & \\
\hline Solo alguna vez & 10 & 4 & 5 & \\
\hline Nunca & 5 & 4 & 5 & \\
\hline \multicolumn{5}{|l|}{ Frecuencia de aplicación de fuerza } \\
\hline Siempre & 17 & 17 & 13 & 0,149 \\
\hline Muchas veces & 11 & 12 & 19 & \\
\hline Algunas veces & 19 & 10 & 10 & \\
\hline Solo alguna vez & 1 & 6 & 4 & \\
\hline Nunca & 7 & 5 & 3 & \\
\hline \multicolumn{5}{|l|}{ Exposición a ruido } \\
\hline Continua & 34 & 21 & 29 & 0,079 \\
\hline Intermitente & 8 & 18 & 7 & \\
\hline Momentánea y fuerte (impacto) & 6 & 7 & 9 & \\
\hline No se percibe ruido & 7 & 4 & 4 & \\
\hline \multicolumn{5}{|l|}{ Tiempo en la posición corporal más frecuente } \\
\hline De 10 a 30 minutos & 17 & 21 & 22 & 0,085 \\
\hline Más de 30 minutos a 2 horas & 8 & 3 & 7 & \\
\hline De 2 a 5 horas & 5 & 12 & 5 & \\
\hline Más de 5 horas & 23 & 14 & 15 & \\
\hline Labor con proyección de partículas hacia la cara & 31 & 40 & 33 & $0,037^{*}$ \\
\hline Uso o almacenamiento de productos inflamables & 14 & 12 & 22 & $0,047^{*}$ \\
\hline
\end{tabular}

* Prueba exacta de Fisher; ** prueba de Kruskal-Wallis; ${ }^{*}$ valor mediana (mínimo y máximo)

Con la matriz construida para explorar la exposición acumulada (cuadro 3), en la que los valores de cero correspondían a los trabajadores que nunca habían hecho labores subterráneas, se observó que entre los cuartiles había diferencias en las variables sociodemográficas y de exposición ocupacional. Por ejemplo, se evidenció que las mujeres llevaban muy poco tiempo laborando en las minas o apenas recientemente habían comenzado a trabajar en ellas. Con relación a las exposiciones, se observó 
Cuadro 3. Condiciones de trabajo percibidas por los mineros de acuerdo con los cuartiles de la matriz de exposición ocupacional en la mina de Guachetá, Colombia, 2012

\begin{tabular}{|c|c|c|c|c|c|}
\hline \multirow[t]{2}{*}{ Variables } & \multicolumn{4}{|c|}{ Matriz de experiencia-ocupación subterránea" } & \multirow[t]{2}{*}{$\mathbf{p}$} \\
\hline & $0(n=48)$ & 1 a $4(n=36)$ & 5 a $8(n=33)$ & 9 a $35(n=37)$ & \\
\hline \multicolumn{6}{|l|}{ Edad (años cumplidos) ${ }^{¥}$} \\
\hline 18 a 27 & $22,5(18-27)$ & $23(18-27)$ & $25(23-27)$ & $27(27-27)$ & $0,0001^{* *}$ \\
\hline 28 a 31 & $29(28-31)$ & $29(28-31)$ & $28,5(28-30)$ & $30(28-31)$ & \\
\hline 32 a 41 & $39(34-40)$ & $36(34-41)$ & $37(32-41)$ & $37(32-41)$ & \\
\hline 42 a 77 & $51(43-64)$ & $45(42-52)$ & 0 & $51,5(42-77)$ & \\
\hline Sexo: hombres & 40 & 36 & 33 & 37 & $<0,001^{\star}$ \\
\hline \multicolumn{6}{|c|}{ Frecuencia de exposición a tóxicos en aire } \\
\hline Siempre & 18 & 18 & 18 & 15 & 0,010 \\
\hline Muchas veces & 3 & 3 & 2 & 12 & \\
\hline Algunas veces & 15 & 7 & 7 & 3 & \\
\hline Solo alguna vez & 0 & 2 & 2 & 1 & \\
\hline Nunca & 12 & 6 & 4 & 6 & \\
\hline \multicolumn{6}{|c|}{ Frecuencia de trabajo habitual de rodillas } \\
\hline Siempre & 2 & 2 & 2 & 6 & 0,040 \\
\hline Muchas veces & 4 & 4 & 5 & 7 & \\
\hline Algunas veces & 6 & 12 & 10 & 12 & \\
\hline Solo alguna vez & 8 & 5 & 7 & 4 & \\
\hline Nunca & 28 & 13 & 9 & 8 & \\
\hline \multicolumn{6}{|c|}{ Frecuencia de manipulación de cargas } \\
\hline Siempre & 13 & 20 & 16 & 10 & 0,005 \\
\hline Muchas veces & 9 & 2 & 7 & 9 & \\
\hline Algunas veces & 10 & 7 & 5 & 13 & \\
\hline Solo alguna vez & 6 & 7 & 3 & 3 & \\
\hline Nunca & 10 & 0 & 2 & 2 & \\
\hline \multicolumn{6}{|c|}{ Frecuencia de movimientos repetitivos } \\
\hline Siempre & 20 & 24 & 14 & 20 & 0,022 \\
\hline Muchas veces & 9 & 5 & 12 & 8 & \\
\hline Algunas veces & 6 & 6 & 6 & 6 & \\
\hline Solo alguna vez & 6 & 0 & 0 & 1 & \\
\hline Nunca & 7 & 1 & 1 & 2 & \\
\hline \multicolumn{6}{|c|}{ Frecuencia de aplicación de fuerza } \\
\hline Siempre & 8 & 15 & 10 & 14 & $<0,001$ \\
\hline Muchas veces & 12 & 7 & 8 & 15 & \\
\hline Algunas veces & 15 & 13 & 8 & 3 & \\
\hline Solo alguna vez & 1 & 1 & 5 & 4 & \\
\hline Nunca & 12 & 0 & 2 & 1 & \\
\hline \multicolumn{6}{|c|}{$\begin{array}{l}\text { Frecuencia de disponibilidad de espacio } \\
\text { para hacer los movimientos necesarios }\end{array}$} \\
\hline Siempre & 33 & 18 & 15 & 17 & $0,008^{*}$ \\
\hline Muchas veces & 4 & 2 & 5 & 8 & \\
\hline Algunas veces & 11 & 9 & 4 & 7 & \\
\hline Solo alguna vez & 0 & 5 & 7 & 5 & \\
\hline Nunca & 0 & 2 & 2 & 0 & \\
\hline \multicolumn{6}{|c|}{ Frecuencia de iluminación adecuada } \\
\hline Siempre & 34 & 20 & 14 & 27 & $0,025^{*}$ \\
\hline Muchas veces & 8 & 4 & 4 & 4 & \\
\hline Algunas veces & 6 & 5 & 7 & 3 & \\
\hline Solo alguna vez & 0 & 4 & 6 & 3 & \\
\hline Nunca & 0 & 3 & 2 & 0 & \\
\hline \multicolumn{6}{|l|}{ Exposición al ruido } \\
\hline No está expuesto & 5 & 3 & 1 & 0 & 0,028 \\
\hline Menos de ocho horas & 19 & 21 & 18 & 21 & \\
\hline Ocho horas & 12 & 11 & 12 & 13 & \\
\hline Más de ocho horas & 12 & 1 & 2 & 3 & \\
\hline \multicolumn{6}{|c|}{ Fuente percibida como más ruidosa } \\
\hline Compresores & 6 & 1 & 3 & 1 & $<0,001$ \\
\hline Equipo de perforación & 4 & 11 & 6 & 4 & \\
\hline Malacate & 16 & 0 & 1 & 0 & \\
\hline Picado de carbón & 0 & 0 & 0 & 1 & \\
\hline Camiones y tractores & 1 & 0 & 0 & 0 & \\
\hline Ventilador & 9 & 6 & 2 & 3 & \\
\hline Martillo & 12 & 18 & 21 & 28 & \\
\hline
\end{tabular}




\begin{tabular}{|c|c|c|c|c|c|}
\hline \multirow[t]{2}{*}{ Variables } & \multicolumn{4}{|c|}{ Matriz de experiencia-ocupación subterránea" } & \multirow[t]{2}{*}{$\mathbf{p}$} \\
\hline & $0(n=48)$ & 1 a $4(n=36)$ & 5 a $8(n=33)$ & 9 a $35(n=37)$ & \\
\hline \multicolumn{6}{|c|}{ Área libre por trabajador $>2 \mathrm{~m}$} \\
\hline Sí & 24 & 16 & 12 & 11 & 0,014 \\
\hline No & 23 & 15 & 16 & 14 & \\
\hline No se ingresó a la mina & 1 & 5 & 5 & 12 & \\
\hline \multicolumn{6}{|l|}{ Altura de techo $<2 \mathrm{~m}$} \\
\hline Sí & 25 & 17 & 18 & 21 & 0,001 \\
\hline No & 22 & 14 & 10 & 4 & \\
\hline No se ingresó a la mina & 1 & 5 & 5 & 12 & \\
\hline \multicolumn{6}{|l|}{ Ancho de pasillo $<1 \mathrm{~m}$} \\
\hline Sí & 0 & 0 & 1 & 0 & 0,004 \\
\hline No & 47 & 31 & 27 & 25 & \\
\hline No se ingresó a la mina & 1 & 5 & 5 & 12 & \\
\hline
\end{tabular}

* Prueba exacta de Fisher; ** prueba de Kruskal-Wallis; ${ }^{*}$ valor mediana (mínimo y máximo); " cuartiles de los valores de la matriz de exposición, incluida la exposición en la mina actual y en las anteriores

la tendencia a encontrar una mayor exposición entre los trabajadores con menos tiempo de vinculación y dedicados a labores subterráneas (valores 1 a 4 de la matriz); como excepciones, se registraron la exposición a sustancias tóxicas en el aire y el trabajo habitual de rodillas.

En el cuadro 4 se muestra la distribución de los problemas de salud reportados por los propios trabajadores. Como se puede apreciar, los dolores lumbares y en los miembros superiores e inferiores, fueron los más frecuentes (más del $30 \%$ ), seguidos por los problemas respiratorios y auditivos. El dolor lumbar resultó ser el más importante, pues fue reportado por cerca de la mitad de los trabajadores. También, se apreció que la suma de las enfermedades diagnosticadas por un médico correspondió a casi una quinta parte del total reportado.

En el cuadro 5 se resumen algunas de las asociaciones bivariadas entre las condiciones de trabajo y el reporte de dolor lumbar, en miembros superiores o en miembros inferiores. Se pudo

Cuadro 4. Prevalencia de los problemas de salud reportados por los trabajadores de las minas de carbón estudiadas

\begin{tabular}{lccc}
\hline Evento reportado & $\mathbf{n}$ & $\%$ & IC $_{95 \%}$ \\
\hline Dolor lumbar & 71 & 46,10 & $38,05-54,31$ \\
Dolor en miembros superiores & 62 & 40,26 & $32,45-48,46$ \\
Dolor en miembros inferiores & 53 & 34,42 & $26,96-42,49$ \\
Trastornos respiratorios & 27 & 17,53 & $11,88-24,47$ \\
Problemas auditivos & 21 & 13,64 & $8,64-20,09$ \\
Enfermedad osteomuscular & 13 & 8,44 & $4,57-14,00$ \\
diagnosticada & & & \\
Signos radiológicos de & 13 & 8,44 & $4,57-14,00$ \\
neumoconiosis & & & \\
Alteración auditiva diagnosticada & 6 & 3,90 & $0,01-8,29$ \\
Neumonía diagnosticada & 4 & 2,60 & $0,71-6,52$ \\
\hline
\end{tabular}

observar que la edad, el sexo masculino, el tipo de jornada de trabajo, el lugar de trabajo y la frecuencia con la que se hacían algunos movimientos o se asumían algunas posturas, se asociaban con uno o varios de estos problemas de salud. Resulta interesante observar que no hubo un patrón evidente que asociara el reporte de los problemas hecho por los propios trabajadores con la percepción de las condiciones de trabajo.

En el cuadro 6 se exploraron algunas condiciones de trabajo mediante regresiones de Poisson ajustadas, y se observó que algunos factores mantenían su asociación y otros dejaban de tenerla cuando se consideraban las demás variables al mismo tiempo. Resaltan las siguientes asociaciones establecidas, con una relación de dosis-respuesta: entre el dolor lumbar y la poca frecuencia de la posición inclinada y el espacio para moverse; el dolor en miembros superiores y el estar sentado de forma habitual y la manipulación de cargas; y el dolor de miembros inferiores y la exposición a vibraciones y los espacios con alturas menores de dos metros.

\section{Discusión}

En este estudio se determinaron y cuantificaron los principales problemas de salud percibidos por los mineros carboníferos de Guachetá; desde su perspectiva, los problemas osteomusculares fueron los más frecuentes, seguidos de los trastornos respiratorios y los auditivos. Asimismo, el estudio permitió identificar algunas condiciones de trabajo que se percibían como asociadas con los trastornos osteomusculares. En general, se pudo observar que las condiciones de trabajo en la minería subterránea del carbón, son precarias y que el reporte que de ellas hacen los propios 
Cuadro 5. Razones de prevalencia crudas de los problemas osteomusculares (dolor) entre mineros del carbón de Guachetá, Colombia, 2012

\begin{tabular}{|c|c|c|c|c|c|c|}
\hline \multirow[t]{2}{*}{ Variables } & \multicolumn{2}{|c|}{ Lumbar } & \multicolumn{2}{|c|}{ Miembros superiores } & \multicolumn{2}{|c|}{ Miembros inferiores } \\
\hline & RP & $\mathrm{IC}_{95 \%}$ & $\mathbf{R P}$ & IC $_{95 \%}$ & $\mathbf{R P}$ & $\mathrm{IC}_{95 \%}$ \\
\hline Edad (años cumplidos) & 1,03 & $1,01-1,04$ & & & & \\
\hline Sexo: hombres & 3,43 & $1,88-6,24$ & & & & \\
\hline Trabajo en minería subterránea & & & & & 0,85 & $0,73-1,00$ \\
\hline \multicolumn{7}{|l|}{ Jornada u horario de trabajo } \\
\hline Jornada partida: mañana y tarde & 1 & & & & 1 & \\
\hline Turno fijo: jornada continua matutina & 2,09 & $1,16-3,75$ & & & 1,15 & $0,62-2,15$ \\
\hline Turno fijo: jornada continua vespertina & 3,75 & $1,73-8,11$ & & & 2,58 & $1,18-5,59$ \\
\hline Turnos rotatorios con horario nocturno & 1,92 & $1,06-3,48$ & & & 1,02 & $0,52-1,99$ \\
\hline Horario irregular & 2,18 & $1,23-3,85$ & & & 1,42 & $0,82-2,45$ \\
\hline \multicolumn{7}{|l|}{ Lugar de trabajo habitual } \\
\hline Al aire libre & 1 & & & & & \\
\hline Cerrado & 1,93 & $1,06-3,52$ & & & & \\
\hline Parcialmente cerrado & 2,05 & $1,06-3,98$ & & & & \\
\hline \multicolumn{7}{|l|}{ Frecuencia de manipulación de cargas } \\
\hline Siempre & & & 1 & & 1 & \\
\hline Muchas veces & & & 1,33 & $0,73-2,43$ & 1,72 & $1,14-2,58$ \\
\hline Algunas veces & & & 0,83 & $0,42-1,67$ & 1,16 & $0,68-1,96$ \\
\hline Solo alguna vez & & & 0,60 & $0,26-1,34$ & 1,03 & $0,52-2,06$ \\
\hline Nunca & & & 1,98 & $1,00-3,94$ & 0,75 & $0,37-1,50$ \\
\hline \multicolumn{7}{|l|}{ Frecuencia de movimientos repetidos } \\
\hline Siempre & 1 & & & & & \\
\hline Muchas veces & 0,69 & $0,42-1,11$ & & & & \\
\hline Algunas veces & 1,58 & $1,00-2,49$ & & & & \\
\hline Solo alguna vez & 0,35 & $0,06-2,00$ & & & & \\
\hline Nunca & 0,38 & $0,15-0,95$ & & & & \\
\hline \multirow{2}{*}{\multicolumn{7}{|c|}{ Frecuencia de disponibilidad de espacio }} \\
\hline & \multicolumn{6}{|c|}{ para una adecuada movilidad } \\
\hline Siempre & & & 1 & & 1 & \\
\hline Muchas veces & & & 0,57 & $0,30-1,09$ & 1,40 & $0,69-2,84$ \\
\hline Algunas veces & & & 1,66 & $0,92-2,96$ & 0,74 & $0,47-1,19$ \\
\hline Solo alguna vez & & & 1,45 & $0,79-2,65$ & 2,12 & $1,29-3,49$ \\
\hline Nunca & & & 1,92 & $0,80-4,60$ & 0,99 & $0,54-1,84$ \\
\hline \multicolumn{7}{|l|}{ Frecuencia de iluminación adecuada } \\
\hline Siempre & 1 & & & & 1 & \\
\hline Muchas veces & 1,95 & $1,25-3,05$ & & & 0,88 & $0,47-1,64$ \\
\hline Algunas veces & 1,11 & $0,65-1,90$ & & & 1,68 & $1,02-2,78$ \\
\hline Solo alguna vez & 1,17 & $0,67-2,04$ & & & 1,16 & $0,68-1,99$ \\
\hline Nunca & 2,97 & $1,80-4,87$ & & & 1,29 & $0,78-2,14$ \\
\hline \multicolumn{7}{|l|}{ Altura de techo superior a 2 metros } \\
\hline Sí & 0.64 & $0.42-0.98$ & & & & \\
\hline No & 1 & & & & & \\
\hline
\end{tabular}

$\mathrm{RP}$ : razón de prevalencia

¥ Solo se presentan las variables en las que, por lo menos, una categoría mostró asociaciones estadísticamente significativas.

trabajadores no siempre facilita la identificación de los patrones dosis-respuesta; solo en algunas condiciones se evidenció este patrón, lo cual puede explicarse por la percepción subjetiva de las condiciones de trabajo y los problemas de salud.

En algunos estudios hechos en Colombia, se han obtenido resultados similares, lo que permite validar los que aquí se presentan. Las características sociodemográficas de los mineros de Guachetá son similares a las de otras regiones en donde se han llevado a cabo dichos estudios $(5,6,10,11,16$ -
22), lo que permite inferir que los atributos exigidos o buscados por los reclutadores de los trabajadores son los mismos sin importar el lugar.

Las asociaciones observadas entre las condiciones de trabajo y de salud, indican que los trabajadores tienden a identificar como riesgosas aquellas condiciones que pueden desembocar en un accidente o un dolor inmediato; las condiciones que requieren un mayor periodo de latencia entre la exposición y la aparición de su efecto, o una exposición acumulada durante periodos prolongados, como la exposición 
Cuadro 6. Razones de prevalencia ajustadas por condiciones de trabajo y problemas osteomusculares entre mineros del carbón de Guachetá, Colombia, 2012

\begin{tabular}{|c|c|c|c|c|c|c|}
\hline \multirow[t]{2}{*}{ Variables } & \multicolumn{2}{|c|}{ Dolor lumbar } & \multicolumn{2}{|c|}{ Dolor de miembro superior } & \multicolumn{2}{|c|}{ Dolor de miembro inferio } \\
\hline & $\mathbf{R P}$ & IC $_{95 \%}$ & $\mathbf{R P}$ & IC $_{95 \%}$ & $\mathbf{R P}$ & IC $_{95 \%}$ \\
\hline \multicolumn{7}{|c|}{ Exposición a vibraciones } \\
\hline Siempre & 1 & & 1 & & 1 & \\
\hline Muchas veces & 1,18 & $0,57-2,48$ & 0,49 & $0,27-0,91$ & 0,78 & $0,47-1,28$ \\
\hline Algunas veces & 1,19 & $0,65-2,19$ & 1,13 & $0,78-1,63$ & 0,48 & $0,29-0,77$ \\
\hline Nunca & 0,70 & $0,34-1,47$ & 0,68 & $0,44-1,06$ & 0,44 & $0,26-0,73$ \\
\hline \multicolumn{7}{|c|}{ Posición habitual: de pie } \\
\hline Siempre & 1 & & 1 & & 1 & \\
\hline Muchas veces & 0,58 & $0,29-1,15$ & 0,36 & $0,22-0,59$ & 0,38 & $0,21-0,66$ \\
\hline Solo alguna vez & 0,38 & $0,09-1,57$ & 0,33 & $0,13-0,81$ & 0,97 & $0,52-1,82$ \\
\hline \multicolumn{7}{|c|}{ Posición habitual: sentado } \\
\hline Siempre & 1 & & 1 & & 1 & \\
\hline Algunas veces & 1,11 & $0,37-3,32$ & 3,47 & $1,25-9,61$ & 1,11 & $0,55-2,22$ \\
\hline Solo alguna vez & 1,05 & $0,35-3,15$ & 3,22 & $1,16-8,93$ & 1,08 & $0,54-2,15$ \\
\hline \multicolumn{7}{|c|}{ Posición habitual: inclinado } \\
\hline Siempre & 1 & & 1 & & 1 & \\
\hline Muchas veces & 0,92 & $0,47-1,80$ & 0,49 & $0,27-0,88$ & 0,79 & $0,44-1,43$ \\
\hline Solo alguna vez & 0,16 & $0,04-0,68$ & 0,48 & $0,25-0,91$ & 0,68 & $0,35-1,34$ \\
\hline Nunca & 0,53 & $0,29-0,99$ & 0,84 & $0,57-1,24$ & 1,04 & $0,66-1,63$ \\
\hline \multicolumn{7}{|c|}{ Manipulación de carga } \\
\hline Sí & 0,64 & $0,25-1,63$ & 1,91 & $1,23-2,96$ & 0,69 & $0,34-1,39$ \\
\hline No & 1 & & 1 & & 1 & \\
\hline \multicolumn{7}{|c|}{$\begin{array}{l}\text { Frecuencia de disponibilidad de espacio } \\
\text { para una adecuada movilidad }\end{array}$} \\
\hline Siempre & 1 & & 1 & & 1 & \\
\hline Algunas veces & 1,44 & $0,82-2,50$ & 1,86 & $1,31-2,64$ & 1,80 & $1,21-2,68$ \\
\hline Solo alguna vez & 0,86 & $0,37-1,98$ & 1,23 & $0,75-2,01$ & 2,06 & $1,29-3,28$ \\
\hline Nunca & 2,58 & $1,06-6,26$ & 2,94 & $1,66-5,19$ & 0,99 & $0,36-2,76$ \\
\hline \multicolumn{7}{|c|}{ Altura de techo superior a 2 metros } \\
\hline Sí & 0,79 & $0,47-1,30$ & 0,74 & $0,53-1,04$ & 0,60 & $0,42-0,86$ \\
\hline No & 1 & & 1 & & 1 & \\
\hline
\end{tabular}

RP: razón de prevalencia

al polvo dentro de la mina, no fueron percibidas como riesgosas. Esto implica que las posibles acciones deben diferenciarse según el problema $y$, sobre todo, deben responder a la necesidad de concientizar a los trabajadores sobre los peligros silentes con efectos a largo plazo, como son los problemas respiratorios.

Los resultados del estudio, además del análisis de los tipos de trabajo en las minas, sugieren que las posturas asociadas con los síntomas en los miembros superiores son el estar de pie o sentado y con los brazos en alto para sostener el martillo neumático, cuyo peso aproximado es de 7 a $10 \mathrm{~kg}$, y empujarlo con fuerza considerable en dirección al manto horizontal superior o techo de la mina con el fin de perforar. Cabe señalar, sin embargo, que debido al peso del equipo y a la vibración que se experimenta en esta tarea, varios trabajadores indicaron que evitaban este tipo de movimientos; además, estas alteraciones posturales y la consecuente presencia de hombro caído, se estudiaron solamente en las minas que tenían manto horizontal (23). El levantamiento de cargas con peso de 10 a $50 \mathrm{~kg} o$ más, la restricción en algunos movimientos debido al reducido espacio de trabajo, lo que obliga a la torsión, flexión y lateralización del tronco, así como las tareas repetitivas y el esfuerzo, se asociaron con el dolor lumbar y en miembros inferiores, cuya atención demanda mayores recursos, como ya se ha indicado en otros estudios (23).

Otro componente de las labores que llevan a cabo los frenteros, piqueros y cocheros, es su asociación con un evidente 'descuento temporal', fenómeno psicológico por el cual los efectos que solo se evidencian en el futuro lejano se perciben como menos significativos que los efectos inmediatos (41). Puesto que este es un rasgo personal asociado con tendencias como el abuso del alcohol y de las drogas, con la obesidad y el juego patológico (42), su identificación puede desempeñar un papel fundamental durante el examen de ingreso al trabajo. 
Los hallazgos de este estudio deben interpretarse teniendo en cuenta sus limitaciones y su enfoque basado en la percepción de los propios trabajadores. El estudio fue transversal y, por lo tanto, la temporalidad entre las exposiciones y los eventos no está garantizada; además, las asociaciones observadas podrían estar mediadas por problemas metodológicos, principalmente el sesgo de selección. En este caso, es posible que se haya presentado el efecto del "trabajador sano", que supone una subestimación de la asociación (43). Este tipo de problemas es frecuente en los estudios de salud ocupacional en el sector minero $(44,45)$. Por otra parte, el empleo del reporte de los propios sujetos sobre las condiciones de trabajo y de salud como fuente de información, suele verse como problemático para la medición (32), pero en este caso debe entenderse como propio de la aproximación conceptual y metodológica usada, por lo cual no es una debilidad sino un elemento coherente con el método adoptado.

En conclusión, los hallazgos de este estudio resaltan la importancia que los mineros le dieron a los trastornos osteomusculares, por encima de la que le otorgaron a los problemas respiratorios y auditivos, lo que contrasta con el hecho de que estos últimos suelen ser los primeros en los que los expertos piensan, puesto que su estudio constituyó un hito en la historia de la salud ocupacional (46). Esta notoria diferencia entre legos y expertos resulta fundamental a la hora de plantear acciones para enfrentar los problemas de la exposición a las condiciones anotadas. En poblaciones como la estudiada se pueden implementar acciones que mitiguen los trastornos osteomusculares (47), sin que ello genere mayores resistencias. Sin embargo, no deben olvidarse las acciones de comunicación en salud orientadas a brindar a los trabajadores información sobre los problemas respiratorios crónicos y la hipoacusia, lo cual podría facilitar un cambio cultural en torno a la percepción del riesgo. La opción de involucrar a los familiares en el proceso ha sido exitosa en Sudáfrica, ya que su perspectiva sobre la enfermedad y la muerte de los mineros, facilita la aceptación del conocimiento por parte de estos (48).

En otros contextos de mayor desarrollo de la salud ocupacional, los resultados podrían explicarse por los cambios recientes introducidos con el uso de tecnologías más avanzadas que implican mejoras en la seguridad de los trabajadores (49). Sin embargo, en el caso estudiado es posible que esto se deba más al hecho de que, por diversas razones, los trabajadores tienden a reconocer como riesgosos aquellos eventos con efectos más inmediatos en su vida cotidiana $(50,51)$. Es en este sentido que debe reconocerse tanto el conocimiento experto como el conocimiento lego, de manera que su complementación permita la planificación de intervenciones que aborden los temas evidentes para los trabajadores, sin olvidar los efectos crónicos más silentes que pueden poner en mayor riesgo la vida. La importancia de los estudios que permiten conocer las percepciones de las comunidades, en este caso, los mineros del carbón, logra cada vez mayor reconocimiento en diversos ámbitos de la salud pública (52). El reto ahora es incorporar estos conocimientos como componentes de las acciones de los expertos en salud ocupacional y de quienes ejercen la autoridad en los lugares de trabajo.

\section{Agradecimientos}

A los trabajadores participantes del estudio, a los estudiantes del Semillero de Investigación del programa de Administración de la Seguridad y Salud Ocupacional de la Universidad Militar Nueva Granada y al personal administrativo de las minas participantes.

\section{Conflicto de intereses}

No hubo ninguno.

\section{Financiación}

Los hallazgos aquí presentados hacen parte del proyecto "Diagnóstico y caracterización de las condiciones laborales de cierta población minera del Municipio de Guachetá", patrocinado por el Centro de Investigaciones de la Facultad de Relaciones Internacionales, Estrategia y Seguridad de la Universidad Militar Nueva Granada y la Alcaldía Municipal de Guachetá, Cundinamarca.

\section{Referencias}

1. Morrice E, Colagiuri R. Coal mining, social injustice and health: A universal conflict of power and priorities. Health Place. 2013;19:74-9. http://dx.doi.org/10.1016/j. healthplace.2012.10.006.

2. Wilson A, Cervantes M. Fraser Institute annual survey of mining companies 2013. Vancouver: Fraser Institute; 2014.

3. Willat T, Timson S, Pichard S, Furuvald M, Ávila-Sánchez L. Introduction to Colombian mining. A new opportunity based on political stability and increasing security. Eng Min J. 2011; Dec: 80-6.

4. Ministerio de Minas y Energía. Política Nacional de Seguridad Minera. Bogotá: Ministerio de Minas y Energía; 2009. 
5. Ospina-Díaz JM, Manrique-Abril FG, Guío-Garzón JA. Salud y trabajo: minería artesanal del carbón en Paipa, Colombia. Avances en Enfermería. 2010;28:107-15.

6. Vega AF. Estudio de medición de gases en las minas de carbón del Salitre de Paipa. Tunja: SENA, Regional Boyacá; 2001.

7. Huertas JI, Huertas ME, Izquierdo S, González ED. Air quality impact assessment of multiple open pit coal mines in northern Colombia. J Environ Manage. 2012;93:121-9. http://dx.doi.org/10.1016/j.jenvman.2011.08.007

8. Huertas JI, Camacho DA, Huertas ME. Standardized emissions inventory methodology for open-pit mining areas. Environ Sci Pollut Res Int. 2011;19:2784-94. http://dx.doi. org/10.1007/s11356-012-0778-3

9. Huertas JI, Huertas ME, Solís DA. Characterization of airborne particles in an open pit mining region. Sci Total Environ. 2012;423:39-46. http://dx.doi.org/10.1016/j. scitotenv.2012.01.065

10. León-Mejía G, Espitia-Pérez L, Hoyos-Giraldo LS, Da Silva J, Hartmann A, Henriques JA, et al. Assessment of DNA damage in coal open-cast mining workers using the cytokinesis-blocked micronucleus test and the comet assay. Sci Total Environ. 2011;409:686-91. http://dx.doi. org/10.1016/j.scitotenv.2010.10.049

11. León-Mejía G, Quintana M, Debastiani R, Dias J, EspitiaPérez L, Hartmann A, et al. Genetic damage in coal miners evaluated by buccal micronucleus cytome assay. Ecotoxicol Environ Saf. 2014;107:133-9. http://dx.doi.org/10.1016/j. ecoenv.2014.05.023

12. León G, Pérez LE, Linares JC, Hartmann A, Quintana M. Genotoxic effects in wild rodents (Rattus rattus and Mus musculus) in an open coal mining area. Mutat Res. 2007;630:42-9. http://dx.doi.org/10.1016/j.mrgentox.2007. 02.007

13. Cabarcas-Montalvo M, Olivero-Verbel J, Corrales-Aldana H. Genotoxic effects in blood cells of Mus musculus and Iguana iguana living near coal mining areas in Colombia. Sci Total Environ. 2012;416:208-14. http://dx.doi.org/10.1016/j. scitotenv.2011.11.080

14. Guerrero-Castilla A, Olivero-Verbel J, Marrugo-Negrete J. Heavy metals in wild house mice from coal-mining areas of Colombia and expression of genes related to oxidative stress, DNA damage and exposure to metals. Mutat Res. 2014;762:24-9. http://dx.doi.org/10.1016/j. mrgentox.2013.12.005

15. Coronado-Posada N, Cabarcas-Montalvo M, OliveroVerbel J. Phytotoxicity assessment of a methanolic coal dust extract in Lemna minor. Ecotoxicol Environ Saf. 2013; 95:27-32. http://dx.doi.org/10.1016/j.ecoenv.2013.05.001

16. Abril-Bolaños J, González-Cáceres M. Condiciones de salud y trabajo en la mina de carbón El Samán, Municipio de Sardinata (Norte de Santander) (tesis). Bogotá: Pontificia Universidad Javeriana; 2009.

17. Estrada J, Rendón I, Valero SC. Algunos aspectos socioeconómicos, de salud y de riesgo laboral de los mineros del carbón de los municipios de Amagá y Angelópolis. Rev Fac Nac Salud Pública. 1997;15:11-36.

18. Ospina JM, González NM, Fernández LJ. Evidencia temprana de alteración funcional por exposición respiratoria: minería artesanal del carbón en Paipa, Colombia. Rev Fac Nac Salud Pública 2011;29:445-53.

19. Garrote-Wilches CF, Malagón-Rojas JN, Morgan G, Combariza D, Varona M. Caracterización de las condiciones de salud respiratoria de los trabajadores expuestos a polvo de carbón en minería subterránea en Boyacá, 2013. Salud UIS. 2014;46:237-47.

20. Quiroz-Arcentales I, Hernández-Flórez LJ, AgudeloCalderón CA, Medina K, Robledo-Martínez R, OsorioGarcía SD. Enfermedad y síntomas respiratorios en niños de cinco municipios carboníferos del Cesar, Colombia. Rev Salud Pública. 2013;15:66-79.

21. Rendón O, Iván D. Neumoconiosis en la minería subterránea del carbón, Amagá, 1995. Rev Fac Nac Salud Pública. 1997;14:46-67.

22. Jiménez NM, Abril FG, Díaz JM, Cubaque MA, Villamil EH. Utilidad de las técnicas de espirometría y oximetría en la predicción de alteración pulmonar en trabajadores de la minería del carbón en Paipa-Boyacá. Revista de la Facultad de Medicina. 2009;57:100-10.

23. Velandia EH, Muñoz JJ. Factores de riesgo de carga física y diagnóstico de alteración osteomuscular en trabajos de minas de carbón en el Valle de Ubaté. Rev Cienc Salud. 2004;2:24-32.

24. Keller C, Bostrom A, Kuttschreuter M, Savadori L, Spence A, White M. Bringing appraisal theory to environmental risk perception: A review of conceptual approaches of the past 40 years and suggestions for future research. J Risk Res. 2012;15:237-56.

25. Slovic P. Perception of risk. Science. 1987;236:280-5. http://dx.doi.org/10.1126/science.3563507

26. de Groot JIM, Steg L, Poortinga W. Values, perceived risks and benefits, and acceptability of nuclear energy. Risk Anal. 2013;33:307-17. http://dx.doi.org/10.1111/j.15396924.2012.01845.x

27. Douglas M. La aceptabilidad del riesgo según las ciencias sociales. Barcelona: Paidós Studio; 1996.

28. Brown P. Popular epidemiology and toxic waste contamination: Lay and professional ways of knowing. J Health Soc Behav. 1992;33:267-81.

29. Forget G, Lebel J. An ecosystem approach to human health. Int J Occup Environ Health. 2001;7(Suppl.2):S3-38.

30. Cook WK. Integrating research and action: A systematic review of community-based participatory research to address health disparities in environmental and occupational health in the USA. J Epidemiol Community Health. 2008;62:66876. http://dx.doi.org/10.1136/jech.2007.067645

31. Pacheco-Magaña LE, Idrovo AJ, Arenas-Monreal L, Cortez-Lugo M, Sánchez-Zamorano LM. Validación del auto-reporte de la localización de residuos sólidos con análisis de Procusto en el contexto de una iniciativa comunitaria participativa. Cad Saúde Pública. 2013;29:195201. http://dx.doi.org/10.1590/S0102-311X2013000100022

32. Wacholder S. When measurement errors correlate with truth: Surprising effects of nondifferential misclassification. Epidemiology. 1995;6:157-61.

33. Hegelund A. Objectivity and subjectivity in the ethnographic method. Qual Health Res. 2005;15:647-68. http://dx.doi. org/10.1177/1049732304273933 
34. Pearce N. Traditional epidemiology, modern epidemiology, and public health. Am J Public Health. 1996;86:678-83. http://dx.doi.org/10.2105/AJPH.86.5.678

35. Pagano M, Gauvreau K. Principles of biostatistics. Second edition. Belmont, CA: Duxbury; 2000. p. 332.

36. Benavides FG, Zimmermann M, Campos J, Carmenate L, Báez I, Nogareda C, et al. Conjunto mínimo básico de ítems para el diseño de cuestionarios sobre condiciones de trabajo y salud. Arch Prev Riesgos Labor. 2010;13:13-22.

37. Donoghue AM. Occupational health hazards in mining: An overview. Occup Med (Lond). 2004;54:283-9. http://dx.doi. org/10.1093/occmed/kqh072

38. Seixas NS, Checkoway H. Exposure assessment in industry specific retrospective occupational epidemiology studies. Occup Environ Med. 1995;52:625-33. http://dx.doi. org/10.1136/oem.52.10.625

39. Mehta CR, Patel NR. A network algorithm for performing Fisher's exact test in $\mathrm{r} \times \mathrm{c}$ contingency tables. J Am Stat Ass. 1983;78:427-34. http://dx.doi.org/10.1080/01621459.1 983.10477989

40. Barros AJD, Hirakata VN. Alternatives for logistic regression in cross-sectional studies: An empirical comparison of models that directly estimate the prevalence ratio. BMC Med Res Methodol. 2003;3:21. http://dx.doi.org/10.1186/1471 2288-3-21

41. Bohm G, Pfister HR. Consequences, morality, and time in environmental risk evaluation. J Risk Res. 2005;8:461-75. http://dx.doi.org/10.1080/13669870500064143

42. Odum AL. Delay discounting: Trait variable? Behav Proc. 2011;87:1-9. http://dx.doi.org/10.1016/j.beproc.2011.02.007

43. Farrow A, Reynolds F. Health and safety of the older worker. Occup Med (Lond). 2012;62:4-11. http://dx.doi. org/10.1093/occmed/kqr148
44. Naidoo R, Robins TG, Becklake M, Seixas N, Thompson ML. Cross-shift peak expiratory flow changes are unassociated with respirable coal dust exposure among South African coal miners. Am J Ind Med. 2007;50:992-8. http://dx.doi.org/10.1002/ajim.20513

45. Graber JM, Cohen RA, Basanets A, Stayner LT, Kundiev Y, Conroy L, et al. Results from a Ukrainian-US collaborative study: Prevalence and predictors of respiratory symptoms among Ukrainian coal miners. Am J Ind Med. 2012;55:1099109. http://dx.doi.org/10.1002/ajim.21997

46. Abrams HK. A short history of occupational health. J Public Health Pol. 2001;22:34-80.

47. Piedrahita H. Algunas experiencias de la aplicación de la ergonomía en el sector minero. Rev Cienc Salud. 2014;12:69-76.

48. Banyini AV, Rees D, Gilbert L. "Even if I were to consent, my family will never agree": Exploring autopsy services for posthumous occupational lung disease compensation among mineworkers in South Africa. Glob Health Action. 2013;6:19518. http://dx.doi.org/10.3402/gha.v6i0.19518

49. McPhee B. Ergonomics in mining. Occup Med (Lond). 2004;54:297-303. http://dx.doi.org/10.1093/occmed/kqh071

50. Weber EU. Experience-based and description-based perceptions of long-term risk: Why global warming does not scare us (yet). Climatic Change. 2006;77:103-20. http:// dx.doi.org/10.1007/s10584-006-9060-3

51. Haas EJ, Hoebbel CL, Rost KA. An analysis of trainers' perspectives within an ecological framework: Factors that influence mine safety training processes. Saf Health Work. 2014;5:118-24. http://dx.doi.org/10.1016/j.shaw.2014.06.004

52. Fernández-Niño JA, Idrovo AJ, Giraldo-Gartner V, Molina-León HF. Los dominios culturales de la malaria: una aproximación a los saberes no institucionales. Biomédica. 2014;34:250-9. http://dx.doi.org/10.7705/biomedica.v34i2.1629 